EPJ Web of Conferences 41, 11007 (2013)

DOI: $10.1051 /$ epjconf/20134111007

(C) Owned by the authors, published by EDP Sciences, 2013

\title{
Shaper-based approach to real-time correction of ultrashort pulse phase drifts and transient pulse dispersion measurements
}

\author{
D. Pestov ${ }^{1}$, G. Rasskazov ${ }^{2}$, A. Ryabtsev ${ }^{2}$, I. Pastirk ${ }^{1}$, and M. Dantus ${ }^{1,2}$ \\ ${ }^{1}$ Biophotonic Solutions Inc., 1401 East Lansing Dr., Suite 112, East Lansing, Michigan 48823, USA \\ ${ }^{2}$ Department of Chemistry, Michigan State University, East Lansing, Michigan 48824, USA
}

\begin{abstract}
We demonstrate for the first time active dispersion and amplitude correction in a fiber laser producing sub-45 fs pulses. The approach is based on single-shot second and third order dispersion measurement based on multiphoton intrapulse interference. The same principle is applied to obtain time-resolved measurements of the transient dispersion induced by an intense laser pulse on a silica window.
\end{abstract}

\section{Principle}

Ultrafast laser systems have proven to be particularly adequate for applications such as material ablation and micromachining, nonlinear spectroscopy and sensing, because of their ability to easily deliver peak power densities of $>10^{12} \mathrm{~W} / \mathrm{cm}^{2}$. While these laser sources have experienced great progress in terms of output pulse characteristics, reliability, and size, they are still notoriously complex and sensitive to changes in ambient environment, leading to alignment drifts, deteriorated pulse characteristics, and depreciated performance over time. Applications of femtosecond lasers outside of laser laboratories thus require automated dispersion and amplitude drift compensation in order to maintain optimum performance without human assistance.

It is reasonable to assume that such phase drifts would manifest themselves primarily in the second- and sometimes third-order dispersion (SOD and TOD, respectively) of the original pulse waveform. Here we take advantage of the inherent sensitivity of nonlinear optical processes to phase distortions. We apply the concept of multiphoton intrapulse interference phase scan (MIIPS) [1], in order to determine SOD and TOD from changes in the second harmonic generation (SHG) spectrum of the a reference pulse. Intensity drifts are simply measured and corrected by adjusting the pulseshaper transmission (amplitude) mask. This real-time version of MIIPS (RT-MIIPS) scheme can be used for monitoring and correction of pulse energy and peak power drifts, allowing for optimum unattended ultrafast laser performance over an indefinite period of time.

The principle behind RT-MIIPS is outlined in Figure 1, where a single local SHG maximum is formed by adding a cubic reference phase mask on an otherwise transform limited pulse. Changes in the magnitude and sign of SOD affect the maximum position within the SHG spectrum. By establishing a one-to-one correspondence between the SHG peak position and the amount of SOD through calibration, one can later accurately measure and correct the change in SOD after acquiring a single SHG spectrum. 
(a)

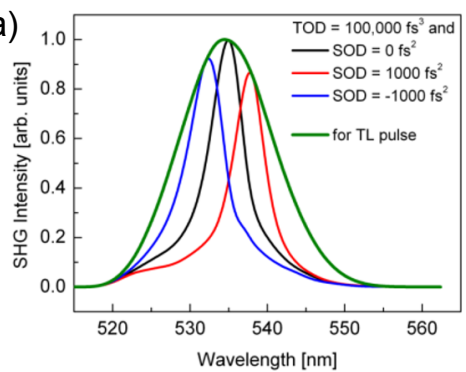

(b)

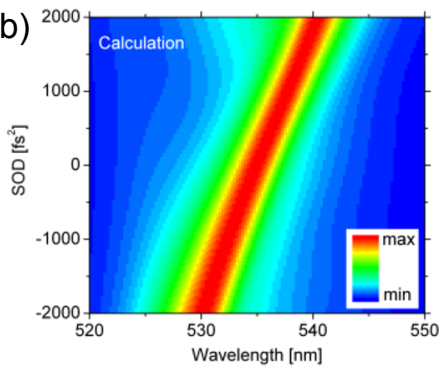

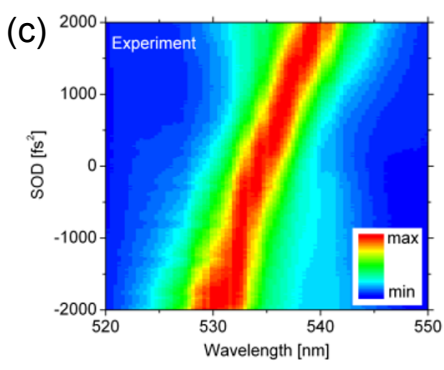

Fig. 1. RT-MIIPS phase correction: (a) The SHG spectra calculated using the experimental laser spectrum from the home-built $\mathrm{Yb}$-doped fiber laser, described in ref. [2]. A cubic phase mask with the TOD of 100,000 $\mathrm{fs}^{3}$ is used as a reference mask. $(b, c)$ The calculated and experimental SHG spectrograms, obtained by scanning the linear chirp. The data is used to calibrate the RT-MIIPS procedure.

\section{Active Phase-and-Amplitude Correction}

We have extended the described approach to account for both SOD and TOD and tested the correction routine in a simple setup, comprising a home-built Yb-doped fiber oscillator [2] and a pulse shaper (MIIPSBox640, Biophotonic Solutions Inc.). An example of validation data is presented in Figure 2.
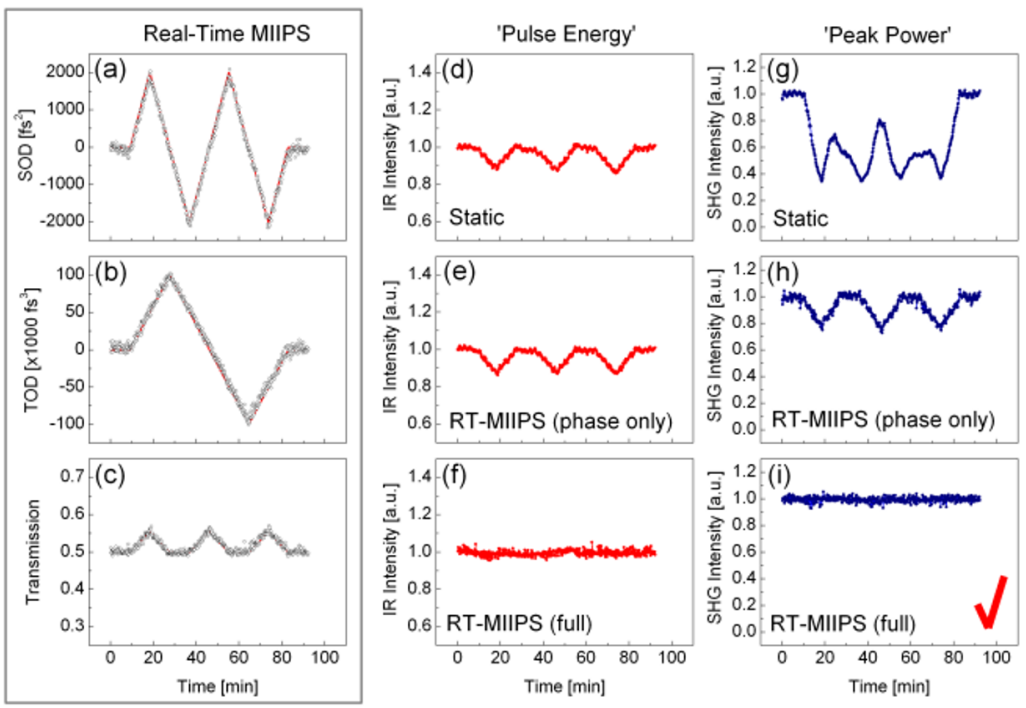

Fig. 2. Validation of RT-MIIPS response to continuous sweeping of SOD, TOD and amplitude: (a) Measured SOD, swept by the shaper within $\pm 2,000 \mathrm{fs}^{2}$; (b) Measured TOD, swept by the shaper within $\pm 100,000 \mathrm{fs}^{3}$; (c) Applied transmission mask. The starting transmission is $50 \%(\mathrm{~T}=0.5)$. The transmission is swept by the shaper by as much as $10 \%$; (d-f) Integrated IR spectra for (d) static phase compensation and transmission $(\mathrm{T} 0=0.5)$ masks, (e) RT-MIIPS corrected phase and static transmission masks, and (f) RT-MIIPS corrected phase and transmission masks. (g-i) Integrated SHG spectra for $(\mathrm{g})$ static phase compensation and transmission $(\mathrm{T} 0=0.5)$ masks, (f) RT-MIIPS corrected phase and static transmission masks, and (i) RT-MIIPS corrected phase and transmission masks. The home-built Yb-doped fiber oscillator is used as a source.

\section{Transient Pulse Dispersion Measurements}

RT-MIIPS can be used for performing time-resolved measurement of transient changes in the dispersion properties of optics. Measurements of transient dispersion changes in fused silica induced by an intense laser pulse are presented here as an example. The results are confirmed by direct comparison with Fourier Transform Spectral Interferometry (FTSI) [3] measurements. 
The pump-probe experimental setup for the two measurements is outlined in Figure 3a. The light from a laser system comprising a Ti:sapphire oscillator, a MIIPSBox640 pulse shaper and a regenerative amplifier ( $800 \mathrm{~nm}$ central wavelength, $1 \mathrm{kHz}$ rep. rate) is split into two beams, denoted in Figure 3a as 'pump' and 'probe'. The probe beam is sent through a computer-controlled delay line. The beams are overlapped on a 2-mm-thick quartz plate. The intensity of the pump beam is set to $2 \times 10^{12} \mathrm{~W} / \mathrm{cm}^{2}$ (average power of $40 \mathrm{~mW}$ ), which is below the damage threshold for fused silica, whereas the 'probe' beam intensity is a few orders of magnitude lower. Pulses are compressed to their transform-limited duration of 40fs using the standard MIIPS technique [1].

For RT-MIIPS measurements, the probe beam after the sample is guided into the RT-MIIPS setup, which encodes a static reference phase and then obtains the resulting SHG spectrum. The experimental data in this case is a 2D spectrogram of SHG signal as a function of the pump-probe delay (Figure 3b). The SHG spectrum shifts are measured and the corresponding SOD values are then plotted as a function of pump-probe delay time (Figure 3c). For FTSI measurements, a Michelson interferometer is used to form two replicas of the probe pulse with 1 ps time separation. The pump pulse arrives at the sample after the first reference probe pulse so that the pump-induced changes affect only the second replica probe. Perturbations induced by the pump pulse lead to a phase change between the replica pulses. The spectral interferometry signal collected by a spectrometer records the changes. The SOD values in this case are extracted through Fourier transform and polynomial fitting of the retrieved phase.
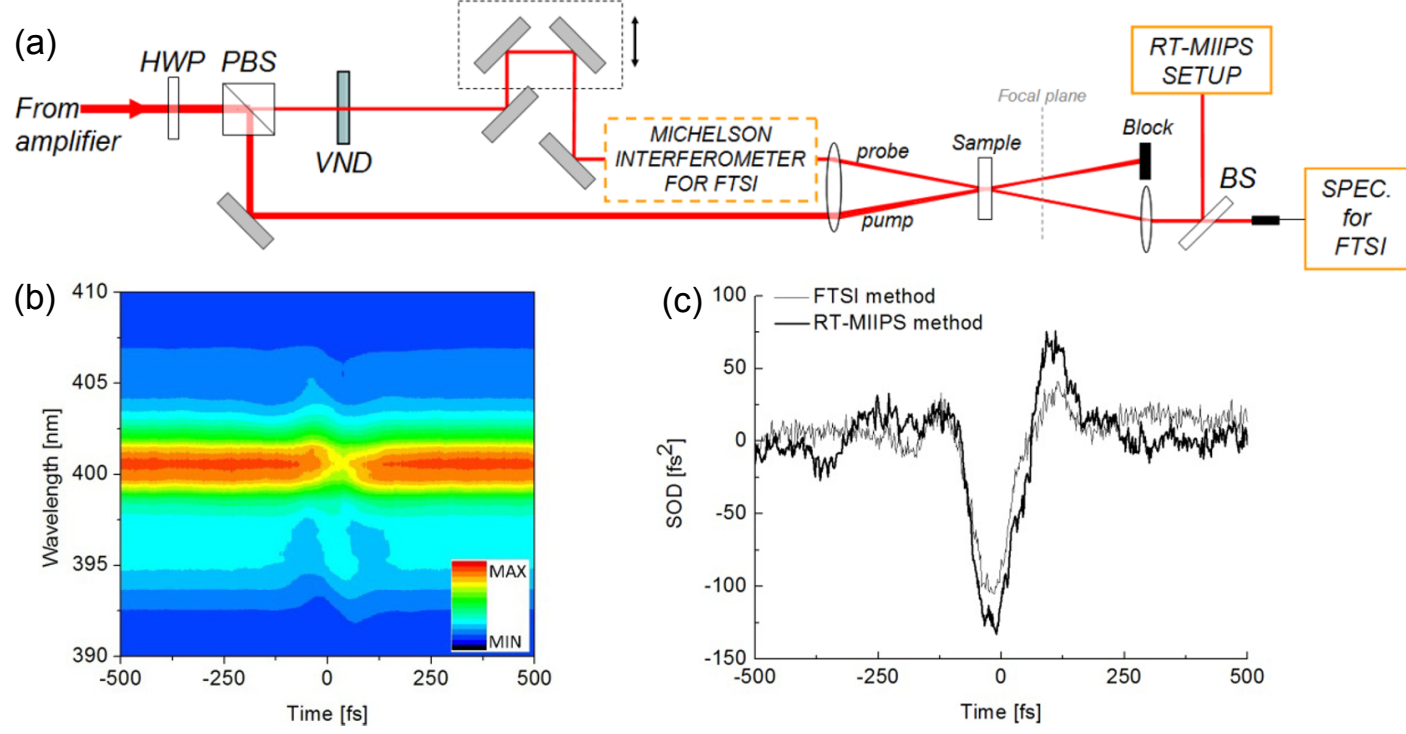

Fig. 3. Experimental setup (a), 2D SHG spectrogram (b) and measured SOD changes in quartz (c). HWP, halfwave plate; PBS, polarizing beam splitter; VND, variable neutral density filter.

Acknowledgements: This work was funded in part by Navy SBIR Program (Contract \# N6833511C-0153) and CHE-1014538 Early-Concept Grant for Exploratory Research from the NSF. We thank Shreya Nad and Nathan Butcher for the early testing of the method.

\section{References}

1. Y. Coello, V. Lozovoy, T. Gunaratne, B. Xu, I. Borukhovich, C. Tseng, T. Weinacht, M. Dantus, J. Opt. Soc. Am. B 25, A140 (2008)

2. B. Nie, D. Pestov, F. Wise, M. Dantus, Optics Express 19, 12074 (2011)

3. P. Audebert, Ph. Daguzan, A. Dos Santos, J. C. Gauthier, J. P. Geindre, S. Guizard, G. Hamoniaux, K. Krastev, P. Martin, G. Petite, and A. Antonetti, PRL 73, 1990 (1994) 